\title{
Extensive und intensive Investition und die Mehrbelastung der Gewinnsteuer
}

\author{
Christian KeuschnigG* \\ Universität St. Gallen (IFF-HSG), CEPR und CESifo
}

April 2007

\begin{abstract}
Zusammenfassung
Um die Auswirkungen der Körperschaftssteuer (Gewinnsteuer) auf Investition und Standortattraktivität zu antizipieren, werden üblicherweise die effektiven Grenzund Durchschnittssteuersätze berechnet. Dieser Beitrag stellt ein Modell monopolistischer Konkurrenz vor und zeigt, wie die extensiven und intensiven Investitionen auf Änderungen in der effektiven Grenz- und Durchschnittsbelastung reagieren. Die intensive Investition meint die marginalen Erweiterungsinvestitionen von etablierten Unternehmen. Die extensive Investition bezieht sich auf die Standortentscheidung für neue Produktionsstätten und resultiert aus der Abwägung zwischen Exporten und Direktinvestitionen als alternative Strategien für den Marktzutritt im Ausland. Das Papier ermittelt die komparativ statischen Effekte der Körperschaftssteuer und zeigt, wie die Mehrbelastung von den Elastizitäten der extensiven und intensiven Investitionsentscheidungen abhängt.

Schlagwörter: Körperschaftssteuer, Exporte, Direktinvestitionen, Mehrbelastung. JEL-Klassifikation: D21, F23, H25, L11, L22.

Adresse: Institut für Finanzwissenschaft und Finanzrecht (IFF-HSG), Universität St.Gallen, Varnbüelstrasse 19, CH-9000 St.Gallen, Schweiz. Tel.: +41-71-224-2520, Fax: -2670, Email: christian.keuschnigg@unisg.ch, http://www.iff.unisg.ch
\end{abstract}

*Ich danke den Teilnehmern der Tagung des Finanzwissenschaftlichen Ausschusses des Vereins für Socialpolitik in Giessen, 8.-10. Juni 2006, sehr herzlich für anregende Diskussionsbeiträge. 


\section{Einleitung}

Die zunehmende internationale Mobilität der Unternehmen im Zuge der Globalisierung haben Standortattraktivität und internationale Wettbewerbsfähigkeit zu dominierenden Anliegen der Steuerpolitik gemacht. Wirtschaftspolitiker geben der Schaffung günstiger steuerlicher Rahmenbedingungen für international mobile und global operierende Unternehmen höchste Priorität. Sie sehen darin eine ganz wesentliche Voraussetzung für Wachstum und Wohlstand im Inland, da die international tätigen Firmen im Vergleich zu den lokalen Unternehmen besonders produktiv und rentabel sind. Direktinvestitionen sind daher eine wichtige Quelle für Beschäftigung und Einkommen im Inland. Die gängige Vorstellung ist, dass eine niedrige Durchschnittsbelastung entscheidend ist, wenn ein Land als Standort für multinationale Direktinvestitionen attraktiver werden möchte. Eine niedrige effektive Durchschnittssteuerbelastung (effective average tax rate, EATR) im Vergleich zu anderen Ländern hilft, die mobilen Unternehmen im Inland zu halten und den Abfluss von Direktinvestitionen zu reduzieren. Gleichzeitig fördert sie auch die Neigung ausländischer Konzerne, Produktionsstätten vor Ort zu errichten anstatt den heimischen Markt mit Exporten aus dem Ausland zu versorgen. Dabei geht es nicht um marginale Erweiterungsinvestitionen, sondern um diskrete Investitionen in komplette Produktionsstätten. Diese diskreten Investitionen hängen von der Durchschnittsbelastung im Vergleich zu alternativen Standorten ab.

Die intensiven Investitionsentscheidungen, d.h. die Neigung zu Erweiterungsinvestitionen am selben Standort, hängen dagegen von der effektiven Grenzsteuerbelastung (effective marginal tax rate, EMTR) ab. Intensive Investitionen reflektieren die Kapitalbildung durch Expansion und Wachstum etablierter Unternehmen. Im Regelfall werden Steuerreformen und Änderungen in den gesetzlichen Steuersätzen beide Masse gleichzeitig verändern and damit Investitionen sowohl am intensiven als auch am extensiven Rand der gesamtwirtschaftlichen Kapitalbildung beeinflussen. Die umfangreiche Studie der Europäischen Kommission (2001) über die Unternehmensbesteuerung in Europa enthält detaillierte Berechnungen zu verschiedenen Massen der effektiven Grenz- und Durch- 
schnittsbelastung im europäischen und weltweiten Vergleich. Der methodische Stand in der Berechnung von effektiven Steuersätzen ist beispielsweise in Sørensen (2004) zusammengefasst.

In der jüngsten Vergangenheit wurden eine ganze Reihe von Steuerreformvorschlägen vorgestellt, welche ganz wesentlich auf eine Verbesserung der internationalen Wettbewerbsfähigkeit des Steuersystems abstellen. Dazu gehören unter anderem die Vorschläge des Sachverständigenrates et al. (SVR, 2006) und des Wissenschaftlichen Beirates beim Bundesministerium der Finanzen (2004) für Deutschland oder das Konzept einer wachstumsorientierten dualen Einkommensteuer von Keuschnigg (2004) und Keuschnigg und Dietz (2007) für die Schweiz. Sogar in den U.S.A. mit ihrem grossen Binnenmarkt erlangen die internationalen Auswirkungen von Steuerreformen eine zunehmende Bedeutung, wie der jüngste Vorschlag des President's Advisory Panel on Federal Tax Reform (2006) belegt.

Der Vorschlag des Sachverständigenrates et al. (2006, S. 7 ff. und S. 31 ff.) sieht im Wesentlichen eine deutliche Absenkung des Körperschaftssteuersatzes auf 25\% und eine Abgeltungssteuer auf persönliche Kapitalerträge wie Zinsen und Dividenden von ebenfalls $25 \%$ vor. Um der Vorbelastung mit der Körperschaftssteuer Rechnung zu tragen, werden auf Investorebene Dividenden im Ausmass einer Normalrendite des Eigenkapitals von 6\% freigestellt, so dass die Gesamtbelastung dieser Dividendenbestandteile bei $25 \%$ bleibt. Darüber hinausgehende Dividendenerträge unterliegen einer Doppelbesteuerung, die zu einer Gesamtbelastung von überdurchschnittlichen Gewinnen in der Nähe des Spitzensteuersatzes der Einkommensteuer auf Lohneinkünfte führt. Dieses Gestaltungselement soll eine Fehldeklaration von hoch besteuerten Lohneinkommen als niedrig besteuerte Kapitaleinkommen verhindern, die Lohnsteuerbasis schützen und damit eine Grundproblem der dualen Einkommensteuer beseitigen. Die kräftige Absenkung des Körperschaftssteuersatzes mindert die Grenz- und Durchschnittsbelastung auf Unternehmensebene, die Freistellung von "normalen" Dividendenerträgen resultiert in einer deutlichen Entlastung 
auf Investorebene. ${ }^{1}$

\begin{tabular}{|l|c|c|c|c|}
\hline Land & $\begin{array}{c}\text { Körperschaftssteuer } \\
\text { Satz, \% (2005) }\end{array}$ & Rang & $\begin{array}{c}\text { EATR } \\
\text { v.H. }\end{array}$ & Rang \\
\hline Deutschland 2005 & 39,35 & 13 & 36,8 & 12 \\
Deutschland nach DIT & 25,00 & 6 & 23,1 & 5 \\
Finnland & 26,00 & 7 & 25,1 & 6 \\
Frankreich & 34,93 & 11 & 35,7 & 11 \\
Irland & 12,50 & $\mathbf{1}$ & 15,0 & $\mathbf{1}$ \\
Italien & 37,25 & 12 & 32,9 & 10 \\
Niederlande & 31,50 & 10 & 29,2 & 8 \\
Österreich & 25,00 & 6 & 23,6 & 5 \\
Polen & 19,00 & 4 & 17,4 & 3 \\
Schweden & 28,00 & 8 & 25,4 & 7 \\
Slovakei & 19,00 & 4 & 17,2 & 2 \\
Ungarn & 17,71 & 2 & 18,1 & 4 \\
U.K. & 30,00 & 9 & 29,5 & 9 \\
U.S. & 40,75 & $\mathbf{1 4}$ & 40,0 & $\mathbf{1 3}$ \\
\hline
\end{tabular}

Gewinnsteuerlast auf Unternehmensebene. EATR: effektive Durchschnittssteuersätze.

DIT: Duale Einkommensteuer. Quelle: SVR et al. (2006), Tabellen 2* und 3*, S.161-162.

Tabelle 1: Duale Einkommensteuer in Deutschland

Der Bericht enthält eine Reihe von Tabellen, welche die Auswirkungen auf die effektive Durchschnittsbelastung dokumentieren und international vergleichen, und streicht die Vorzüge des Reformvorschlags bezüglich der internationalen Standortattraktivität besonders heraus. Demnach würde die Umsetzung des Konzepts die Position Deutschlands in der internationalen Rangordnung der effektiven Durchschnittsbelastung auf der Unternehmensebene entscheidend verbessern. So würde die vorgeschlagene Steuerreform die Stellung Deutschlands im internationalen Steuerwettbewerb nachhaltig stärken. Tabelle 1 ist dem Gutachten entnommen und soll zeigen, dass sich Deutschland im internationalen Vergleich der Durchschnittsbelastungen der aufgelisteten Länder vom 12. auf den 5. Platz

\footnotetext{
${ }^{1}$ Das Prinzip zur Vermeidung der Umqualifizierung von Lohneinkommen ist ähnlich wie im Vorschlag SDES für eine Schweizerische Duale Einkommensteuer in Keuschnigg (2004) und Keuschnigg und Dietz (2007). Der zentrale Unterschied besteht darin, dass im Konzept SDES die Abzugsfähigkeit einer Normalrendite des Eigenkapitals auf Unternehmensebene bei der Gewinnsteuer und nicht auf Investorebene angesiedelt ist. Der Grund dafür ist die Absicht, noch wesentlich stärker die Standortattraktivität zu verbessern. Die auf internationalen Börsen kotierten, multinationalen Unternehmen berücksichtigen in ihren Standortentscheidungen im Regelfall die Steuern auf Investorebene nicht, sondern stellen hauptsächlich auf den Vergleich der Gewinnsteuerbelastung ab (vgl. dazu auch SVR et al., 2006, Ziffer 5, S. 2-3).
} 
verbessern würde. Es ist interessant festzustellen, dass der Bericht die effektiven Grenzsteuerbelastungen gar nicht mehr explizit ausweist, sondern sich auf die Berechnung der Kapitalnutzungskosten beschränkt. Es bleibt insgesamt der Eindruck, dass das Gutachten der Absenkung der Durchschnittsbelastung eine wesentlich grössere Bedeutung beimisst als der Reduktion der Grenzsteuersätze.

\begin{tabular}{|l|c|c|c|c|}
\hline Land & $\begin{array}{c}\text { Körperschafts } \\
\text { steuersatz }\end{array}$ & $\begin{array}{c}\text { Kapital- } \\
\text { kosten }\end{array}$ & EMTR & EATR \\
\hline Österreich & 34.00 & 6.3 & 20.9 & 29.8 \\
Belgien & 40.17 & 6.4 & 22.4 & 34.5 \\
Dänemark & 32.00 & 6.4 & 21.9 & 28.8 \\
Finnland & 28.00 & 6.2 & 19.9 & 25.5 \\
Frankreich & 40.00 & 7.5 & 33.2 & 37.5 \\
Deutschland & 39.30 & 6.8 & 26,0 & 34.8 \\
Griechenland & 40.00 & 6.1 & 18.2 & 29.6 \\
Irland & 10.00 & 5.7 & 11.7 & 10.5 \\
Italien & 41.25 & 4.8 & -4.1 & 29.8 \\
Luxemburg & 37.45 & 6.3 & 20.7 & 32.2 \\
Niederlande & 35.00 & 6.5 & 22.6 & 31.0 \\
Portugal & 37.40 & 6.5 & 22.5 & 32.6 \\
Spanien & 35.00 & 6.5 & 22.8 & 31.0 \\
Schweden & 28.00 & 5.8 & 14.3 & 22.9 \\
UK & 30.00 & 6.6 & 24.7 & 28.2 \\
\hline
\end{tabular}

Quelle: Europäische Kommission (2001), Tabellen 7 und 8. Nur Unternehmensebene. Deutschland nach 2001, Box 7, Annex Tabelle 1a.

Tabelle 2: Internationaler Vergleich effektiver Steuersaetze

Tabelle 2 fasst einige wichtige Berechnungen der Europäischen Kommission (2001) zu effektiven Steuersätzen in Europa zusammen und enthält auch die Grenzsteuersätze. Für Deutschland sind dabei die Auswirkungen der Steuerreform 2001 berücksichtigt. Mit Ausnahme von Frankreich belastet demnach kein anderes Land der Europäischen Union die Investitionen höher als Deutschland. Ausserdem fällt auf, dass die effektiven Durchschnittssätze deutlich höher liegen als die Grenzsteuerbelastungen. Sie hängen stark von der Höhe der tariflichen Steuersätze ab. Dieser Beitrag geht unter anderem auch der Frage nach, ob die effektive Durchschnittsbelastung tatsächlich das alleinige, relevante Mass für die steuerliche Verzerrung der Standortentscheidungen ist.

Die meisten akademischen Beiträge zur Besteuerung von Direktinvestitionen (vgl. die Übersichtsbeiträge von Gordon und Hines, 2002, Gresik, 2001, Weichenrieder, 1995, und 
Janeba, 1997, oder die Aufsätze von Haufler und Schjelderup, 2000, oder Davies, 2004, um nur einige wenige zu erwähnen) stellen nicht sehr eng auf diese deskriptiven Masse von effektiven Grenz- und Durchschnittssteuersätzen ab. In der Literatur dominiert der Ansatz, wonach Direktinvestitionen solange fliessen, bis das Grenzprodukt des Kapitals nach Steuern an allen Standorten gleich ist. Steuern treiben einen Keil zwischen die gemeinsame Nettoertragsrate und die regionalen Bruttoertragsraten und führen so zu einer ineffizienten, internationalen Allokation des Kapitals. Diese Betrachtungsweise stellt ausschliesslich auf marginale Investitionen in einen bereits vorhandenen Kapitalbestand ab und schliesst die diskrete Natur von Direktinvestitionen aus. Damit ist es unmöglich, die Rolle der Durchschnittsbelastung für die Standortattraktivität eines Landes zu erkennen.

Die empirischen Arbeiten von Devereux und Griffith (1998) und anderen, wie sie jüngst von Devereux, Griffith und Klemm (2002) zusammengefasst wurden, weisen jedoch einen bedeutsamen Einfluss der Durchschnittsbelastung auf die Höhe von Direktinvestitionen nach. Die neuere und noch wenig umfangreiche theoretische Literatur hat daher Modelle von Direktinvestitionen zur Erschliessung ausländischer Märkte mit unvollständigem Wettbewerb entwickelt, mit denen der Einfluss der Besteuerung auf die diskreten Standortentscheidungen herausgearbeitet wird (vgl. Devereux und Hubbard, 2003, Devereux und Griffith, 2003, Fuest, 2005, oder auch Bond, 2000, für eine erste Diskussion). Diese Beiträge ignorieren dagegen die intensiven Investitionen, welche für die immobilen, nationalen Unternehmen weiterhin entscheidend sind.

Diese neue, finanzwissenschaftliche Literatur vermag jedoch nicht, die separate Rolle von Grenz- und Durchschnittsbelastungen für extensive und intensive Investitionen in einem einheitlichen Rahmen zu erklären. Der vorliegende Beitrag füllt diese Lücke. Es ist noch wenig bis gar nicht bekannt, wie die Gewinnsteuer, ${ }^{2}$ indem sie eine bestimmte Höhe der effektiven Steuersätze EATR und EMTR impliziert, die gesamtwirtschaftliche Investitionstätigkeit mehr auf den intensiven oder extensiven Rand lenkt. Noch grösser

\footnotetext{
${ }^{2}$ In der Schweiz wird die Körperschaftssteuer als Gewinnsteuer bezeichnet. Diese Benennung wird in der Folge beibehalten.
} 
ist das Unwissen darüber, wie die Verhaltensanpassungen an diesen Rändern die volkswirtschaftlichen Kosten der Gewinnsteuer beeinflussen. Idealerweise sollte es möglich sein, eine Parallele zur Literatur über die Lohnbesteuerung bei gleichzeitiger Reaktion des intensiven und extensiven Arbeitsangebots zu ziehen (vgl. Saez, 2002, Immervoll, Kleven, Kreiner and Saez, 2007, und Kleven und Kreiner, 2006). Tatsächlich zeigt der vorliegende Aufsatz, dass die Mehrbelastung der Gewinnsteuer in ganz ähnlicher Weise von den Elastizitäten der diskreten und marginalen Investitionen und den Grenz- und Durchschnittssteuersätzen abhängt. Die Ermittlung der Mehrbelastung erfordert allerdings eine konsistente Wohlfahrtsanalyse der Gewinnsteuer auf unvollständigen Märkten. Trotz der grossen steuerpolitischen Bedeutung wurde eine solche Analyse bisher für zu aufwendig und kompliziert gehalten (vgl. die publizierten Kommentare zum Aufsatz von Devereux, Griffith und Klemm, 2002).

Im Vergleich zu bisherigen Ansätzen in der Finanzwissenschaft beschreitet dieser Beitrag einen ganz anderen Weg, um diskrete und marginale Investitionen gleichzeitig zu berücksichtigen. Ausgangspunkt ist die neue Aussenhandelstheorie, welche die Heterogenität im Unternehmenssektor betont. Diese Theorie erklärt, wie Firmen zwischen Exporten und Direktinvestitionen als alternative Strategien zur Belieferung ausländischer Märkte abwägen (vgl. Melitz, 2003, Helpman, 2006, Grossman und Helpman, 2005, Helpman, Melitz und Yeaple, 2004, u.a.). Hier wird eine eigene, stark vereinfachte, probabilistische Version des "Melitz-Modells" mit monopolistischer Konkurrenz im Unternehmenssektor entwickelt. Um die Analyse möglichst weit zu vereinfachen, beschränken wir uns auf abfliessende Direktinvestitionen von heimischen Unternehmen und unterdrücken den Zufluss von Direktinvestitionen. Ebenso schalten wir Produktivitätsunterschiede im Firmensektor aus. Diese mögen für andere Fragestellungen sehr interessant sein, spielen für das hier betrachtete Problem aber nur eine untergeordnete Rolle. Anstatt solcher unterschiedlicher Produktivitäten führt der probabilistische Ansatz ein Erfolgsrisiko des ausländischen Marktzutritts ein, welches zwischen verschiedenen Firmen variiert. Damit kann die Symmetrie der Unternehmen bezüglich aller anderen Charakteristiken erhalten bleiben. So wird die Analyse wesentlich einfacher. 
Nachdem Direktinvestitionen im Vergleich zu Exporten mit zusätzlichen Fixkosten für die Errichtung einer Produktionsstätte im Ausland verbunden sind, zahlen sich Direktinvestitionen nur für die Unternehmen mit der höchsten Erfolgswahrscheinlichkeit für den Marktzutritt im Ausland aus. Für andere Unternehmen, die mit grösseren Schwierigkeiten der Produkteinführung im Ausland rechnen (geringere Erfolgswahrscheinlichkeit), ist der Export trotz der damit verbundenen Transportkosten die billigere Alternative, da sie in diesem Fall die Fixkosten der Etablierung einer separaten ausländischen Produktionsstätte vermeiden können. Die Entscheidung zwischen Exporten und Direktinvestitionen spiegelt somit eine Abwägung zwischen Marktnähe und Konzentration der Produktion wider (proximity concentration trade-off): Direktinvestitionen sparen Transportkosten, duplizieren aber wegen der separaten Produktion im In- und Ausland die Fixkosten der Produktion.

Der Beitrag zeigt zunächst, wie die Gewinnsteuer die intensive Investition und damit das Unternehmenswachstum hemmt, indem sie abhängig von der Höhe des effektiven Grenzsteuersatzes die Kapitalnutzungskosten verteuert. Die variable Anzahl von heimischen Unternehmen, welche Direktinvestitionen den Exporten vorziehen und die Produktion in das Ausland verlagern, definiert den extensiven Rand der gesamtwirtschaftlichen Investition. Danach zeigen wir, wie die Durchschnittsbelastung der heimischen Gewinnsteuer den Unternehmenswert reduziert, der mit der Exportproduktion im Inland erzielt werden kann. Nach der international üblichen Befreiungsmethode kann die heimische Gewinnsteuer den Unternehmenswert von ausländischen Tochtergesellschaften nicht beeinträchtigen. Indem sie einseitig den Unternehmenswert aus der Exportproduktion im Inland reduziert, führt sie zu einer Verlagerung der Produktion ins Ausland und mindert die Investionen am extensiven Rand. Als weitere Innovation charakterisiert das Papier anhand einer expliziten Wohlfahrtsanalyse die Mehrbelastung der Gewinnsteuer und zeigt, wie sie von den extensiven und intensiven Investitionselastizitäten abhängt. Der zweite Abschnitt entwickelt nun den Modellrahmen. Abschnitt 3 ermittelt die komparativ statischen Effekte und Abschnitt 4 charakterisiert die Mehrbelastung der Gewinnsteuer. 


\section{Das Modell}

Wir entwickeln die Argumente anhand eines einfachen Zwei-Perioden-Modells mit monopolistischer Konkurrenz und abfliessenden Direktinvestitionen. In der ersten Periode wird mit einer fixen Arbeitsausstattung ein traditionelles Numeraire-Gut produziert, welches konsumiert oder investiert wird. Mit einer Ricardianischen Technologie wird aus einer Einheit Arbeit eine Einheit Output produziert. Vollständiger Wettbewerb im traditionellen Sektor fixiert den Lohnsatz auf eins. Eine feste Zahl $n$ von innovativen Firmen investiert in der ersten Periode Kapital, um damit in der zweiten Periode ein differenziertes Gut zu produzieren. Jede dieser Firmen besitzt ein weltweit geschütztes Patent für eine spezifische Produktvariante, die ein nahes Substitut für andere Varianten darstellt.

Die Anbieter differenzierter Produkte produzieren unter Bedingungen der monopolistischen Konkurrenz für eine potentiell weltweite Nachfrage. Allerdings ist die Produkteinführung und damit der Marktzutritt im Ausland schwieriger als im Heimatland und unterliegt einem Marktrisiko. Daher werden die Firmen zwar immer den Inlandsmarkt beliefern, der Marktzutritt im Ausland mag jedoch erfolgreich sein oder auch nicht. Im Falle des Scheiterns wird die Produktvariante im Ausland nicht angeboten. Mit dem Versuch, den Auslandsmarkt zu erschliessen, muss das Unternehmen auch entscheiden, ob dieser mit Exporten aus heimischer Produktion verbunden mit zusätzlichen Transportkosten beliefert werden soll. Alternativ könnte sie die Transportkosten auch einsparen, indem sie die Produktion in das Ausland verlagert und lokal vor Ort produziert. Die Errichtung einer ausländischen Tochtergesellschaft ist jedoch mit zusätzlichen administrativen und anderen Fixkosten verbunden. Um das Modell möglichst einfach zu halten, unterdrücken wir die Produktion differenzierter Güter durch ausländische Firmen, so dass im Inland keine Importe oder zufliessenden Direktinvestitionen stattfinden. Das Ausland ist vollständig auf das traditionelle Gut spezialisiert. Der Konsum differenzierter Güter im Ausland stammt ausschliesslich aus Importen oder aus der Produktion inländischer Unternehmen mit ihren Produktionsstätten im Ausland. 


\subsection{Die Nachfrage}

Inländische Haushalte sind mit einem fixen Arbeitsangebot von $L$ ausgestattet. In der ersten Periode erzielen sie daher ein fixes Arbeitseinkommen, konsumieren eine Menge $C_{1}$ des traditionellen Gutes (Numeraire) und sparen das restliche Einkommen. In der zweiten Periode beziehen sie aggregierte Gewinne $\pi^{e}$ aus dem anteiligen Besitz der monopolistischen Firmen. Die Anteile müssen sich wie andere Assets auch mit einer Rendite von $r$ verzinsen. Zusätzlich erhalten die Haushalte pauschale Transfers $z$ aus der Rückerstattung des Steueraufkommens. Die Ausgaben werden auf $C_{2}$ für den Konsum des traditionellen Gutes und $E$ für den Kauf von $n$ differenzierten Gütern aufgeteilt. Die Budgetbeschränkungen für die beiden Perioden lauten

$$
C_{1}=L-S, \quad C_{2}+E=R S+\pi^{e}+z, \quad E=\int_{0}^{n} p_{j} c_{j} d j=n p c .
$$

Die letzte Gleichung spiegelt die Symmetrie der Präferenzen und Kosten der im Inland abgesetzten Produktvarianten wider. Indem man aus der ersten Gleichung für die Ersparnisse in die zweite einsetzt, erhält man die intertemporale Budgetbeschränkung ausgedrückt in Einheiten der zweiten Periode,

$$
R C_{1}+C_{2}+E=L R+\pi^{e}+z, \quad R \equiv 1+r .
$$

Die Präferenzen seien linear separabel, so dass gegenwärtiger und zukünftiger Konsum perfekte Substitute sind. Damit muss der Zinssatz $r$ gleich der fixen Zeitpräferenzrate sein. Die Wohlfahrt der Haushalte hängt daher nicht vom Zeitpunkt, sondern nur von der diskontierten Gesamtmenge des Konsums ab. Solange der Konsumaufschub mit einer Verzinsung $r$ kompensiert wird, wird jeder beliebige Investitionsbedarf angespart. Der Lebensnutzen gemessen in Einheiten der zweiten Periode beträgt $V=R C_{1}+C_{2}+\int_{0}^{n} v\left(c_{j}\right) d j$. Indem man für den Konsum des traditionellen Gutes aus (2.2) einsetzt, folgt

$$
V=L R+\pi^{e}+z+\int_{0}^{n}\left[v\left(c_{j}\right)-p_{j} c_{j}\right] d j .
$$

Die eckige Klammer drückt die Konsumentenrente aus dem Konsum einer innovativen Produktvariante aus. Die Nachfrage folgt aus der Nutzenmaximierung. Bei einer isoelasti- 
schen Spezifikation $v\left(c_{j}\right)=A^{1-\alpha}\left(c_{j}\right)^{\alpha} / \alpha$ mit $0<\alpha<1$ ergibt die notwendige Bedingung $p_{j}=v^{\prime}\left(c_{j}\right)$ eine Nachfragefunktion

$$
c_{j}=A\left(p_{j}\right)^{-\varepsilon}, \quad \varepsilon=1 /(1-\alpha)>1 .
$$

Der Parameter $\varepsilon$ ist die Preiselastizität der Nachfrage.

Das Ausland sei mit einem fixen Arbeitsangebot $L^{f}$ ausgestattet, wobei ausländische Variablen mit dem oberen Index $f$ markiert sind. Die Technologie im Ausland produziert mit einer Investition von einer Einheit des Standardgutes $R$ Einheiten desselben Gutes in der zweiten Periode. Das Ausland sei der Einfachheit halber auf die Produktion des traditionellen Gutes spezialisiert. Der Konsum innovativer Produktvarianten in der zweiten Periode speist sich daher ausschliesslich aus Importen oder aus den Niederlassungen multinationaler Unternehmen, welche aus hereinfliessenden Direktinvestitionen stammen. Nachdem der Markzutritt von fremden Unternehmen mit Risiko behaftet ist, werden nicht alle Varianten im Ausland angeboten, die im Inland verfügbar sind. Die Produktvielfalt im Ausland beträgt $n_{X}+n_{I}<n$, wobei der tief gestellt Index die Varianten bezeichnet, die aus Importen und aus hereinfliessenden Direktinvestitionen stammen. Wegen der Symmetrie innerhalb dieser Produktgruppen lauten die Budgetbeschränkungen des Auslands

$$
C_{1}^{f}=L^{f}-S^{f}, \quad C_{2}^{f}+E^{f}=R S^{f}, \quad E^{f}=n_{X} p_{X} c_{X}+n_{I} p_{I} c_{I} .
$$

Der Lebensnutzen beträgt $V^{f}=R C_{1}^{f}+C_{2}^{f}+\int_{0}^{n^{f}} v\left(c_{j}^{f}\right) d j$ bzw., nach Substitution von (2.5), $V^{f}=R L^{f}+n_{X}\left[v\left(c_{X}\right)-p_{X} c_{X}\right]+n_{I}\left[v\left(c_{I}\right)-p_{I} c_{I}\right]$. Die Nachfrage des Auslands nach einer einzelnen Variante folgt aus der Bedingung $p_{j}^{f}=v^{\prime}\left(c_{j}^{f}\right)$. Abgesehen vom Parameter $A^{f}$, der die Grösse des Auslandsmarktes bestimmt, sei die Spezifikation der Nutzenfunktion identisch mit (2.4). Die ausländische Nachfrage nach Variante $j$ beträgt

$$
c_{j}^{f}=A^{f}\left(p_{j}^{f}\right)^{-\varepsilon}
$$




\subsection{Produktion für den Inlandsmarkt}

Wir stellen hauptsächlich auf Produktion und Handel von differenzierten Gütern des Inlands ab. Um den Inlandsmarkt zu bedienen, investieren die Unternehmen in der ersten Periode $k$ Einheiten des Standardgutes. Abschreibungen seien ausgeschlossen, so dass in der zweiten Periode noch $k$ Einheiten des traditionellen Gutes vorhanden sind. Zusätzlich werden mit den $k$ Einheiten Kapital $k$ Einheiten eines differenzierten Gutes produziert, wobei wir den Variantenindex $j$ unterdrücken, um die Notation übersichtlich zu halten. Eine monopolistische Firma beliefert die gesamte Marktnische für ihre Variante im Inland, $c=k$, und erzielt Erlöse $p k$ in der Höhe der Konsumausgaben in (2.1). Der Staat erhebt eine proportionale Gewinnsteuer mit dem Satz $t$, wobei ein Anteil $e$ der Investitionen in der ersten Periode abzugsfähig sei. Mit $e=1$ könnte die Firma die gesamte Investition sofort abschreiben, so dass die Gewinnsteuer gleich einer investitionsneutralen Cash-Flow Steuer wäre. Hingegen hemmt die Steuer mit $e<1$ die Investitionsneigung. Der Unternehmenswert aus der Belieferung des Inlandsmarktes beträgt $\pi / R$, so dass $\pi$ den auf die zweite Periode aufgezinsten Wert angibt,

$$
\frac{\pi}{R}=\frac{(1-t) p k+(1-e t) k}{R}-(1-e t) k, \quad \pi=(1-t) p k-(1-e t) r k .
$$

Insgesamt fallen Steuereinnahmen von $\pi^{T}=t(p-e r) k$ an.

In der Wahl der optimalen Investition berücksichtigt die Firma ihre Monopolposition $c=k$ in ihrer Marktnische. Die Preis-Absatzkurve in (2.4) ergibt eine Erlösfunktion, die konkav im Kapitaleinsatz ist, ${ }^{3} p(k) k=A^{1-\alpha} k^{\alpha}$. Alternativ können wir mit $k=A p^{-\varepsilon}$ die Erlöse aus dem Inlandsabsatz wie folgt schreiben,

$$
p k=A p^{1-\varepsilon} .
$$

Nach einer geringfügigen Umformulierung von (2.7) erhält man die Investition einer monopolistisch kompetitiven Firma aus

$$
\pi=\max _{k}(1-t)(p k-u k), \quad u \equiv \frac{1-e t}{1-t} r
$$

\footnotetext{
${ }^{3}$ Aus diesem Grund können wir uns auf eine lineare Technologie beschränken. Eine konkave Produktionsfunktion $f(k)$ würde lediglich die Analyse ohne zusätzliche Einsichten verkomplizieren.
} 
wobei $u$ die Kapitalnutzungskosten bezeichnet. Man beachte, dass jeder Outputzuwachs aus einer höheren Investition den erzielbaren Produzentenpreis $p$ verringert. Die Optimalitätsbedingung lautet daher $p-u+k \cdot d p / d k=0$. Die Verwendung der Preiselastizität in (2.4) ergibt

$$
\alpha \cdot p(k)=u, \quad k=A \cdot(\alpha / u)^{\varepsilon}
$$

Wie üblich ist der Preis gleich einem fixen Aufschlag 1/ $\alpha$ über die Einheitskosten, die hier lediglich aus den Kapitalnutzungskosten $u$ bestehen. Die Nachfragekurve (2.4) bestimmt den Absatz, der zu diesem Preis möglich ist, und ergibt damit den optimalen Kapitaleinsatz. Die Grenzerlöskurve $\alpha p(k)$ übernimmt die Rolle einer fallenden Grenzproduktivitätskurve in herkömmlichen Investitionsmodellen, wonach der optimale Kapitaleinsatz durch die Gleichheit von Grenzprodukt und Kapitalnutzungskosten bestimmt ist. Abbildung 1 illustriert graphisch das Investitionsproblem einer monopolistisch kompetitiven Firma, die ihre Investition solange ausdehnt, bis der Grenzerlös gleich den Grenzkosten des Kapitaleinsatzes entspricht.

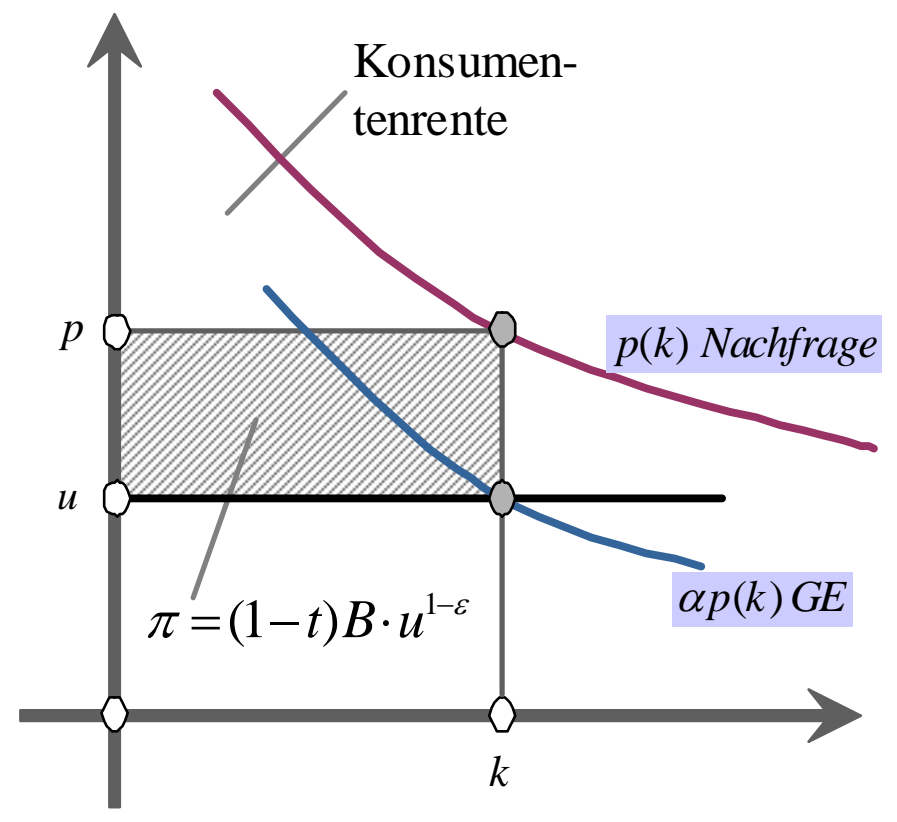

Abb. 1: Investition und Gewinn

Eine geschlossene Form der Gewinnfunktion erhält man, wenn mit der Aufschlagsregel $\alpha p=u$ die Kapitalkosten $u$ in $(2.9)$ ersetzt werden, $\pi=(1-t)(1-\alpha) p k$. Indem man $p k$ 
durch (2.8) ersetzt und noch einmal $p=u / \alpha$ verwendet, folgt

$$
\pi=(1-t) B / u^{\varepsilon-1}, \quad B \equiv(1-\alpha) A \alpha^{\varepsilon-1} .
$$

\subsection{Markzutritt im Ausland}

Eine heimische Unternehmung trifft für ihre differenzierte Produktvariante auf eine weltweite Nachfrage. Angenommen die Markteinführung im Ausland war erfolgreich und die Firma habe sich entschieden, im Inland zu produzieren und den Auslandsmarkt mit Exporten zu beliefern. Exporte verursachen zusätzliche reale Handelskosten $\theta-1$ aus dem Transport und der Abfertigung über die Grenze. Wegen der Transport- und Handelskosten muss ein Exporteur mehr produzieren, als beim ausländischen Nachfrager ankommt, $k_{X}>c_{X}$. Die Differenz entspricht den Handelskosten. Um diese abzudecken, muss der ausländische Konsumentenpreis $p_{X}$ den inländischen Produzentenpreis $p$ um den Faktor $\theta$ übersteigen. Es gilt

$$
p_{X}=\theta p, \quad k_{X}=\theta c_{X}, \quad p_{X} c_{X}=p k_{X}, \quad \theta \geq 1
$$

Wenn eine Firma mit einem Monopol für die Variante $j$ erfolgreich den Export aufnimmt, dann muss sie zusätzlich zum Inlandsabsatz $c=k$ eine Menge $k_{X}$ produzieren. Die entsprechende Investition in der ersten Periode steigert den Firmenwert um den Gewinn $\pi_{X}$ aus dem Exportgeschäft, so dass zusammen mit der Bearbeitung des Heimmarktes ein gesamter Unternehmenswert von $\pi+\pi_{X}$ geschaffen wird. Der Wert des Exportgeschäftes beträgt

$$
\pi_{X}=(1-t) p k_{X}-(1-e t) r k_{X}=(1-t)(p-u) k_{X}
$$

Die Steuerzahlungen in der zweiten Periode belaufen sich auf $\pi_{X}^{T}=t(p-e r) k_{X}$.

Bei einem Nachfragepreis von $p_{X}=\theta p$ beträgt die Auslandsnachfrage in (2.6) $c_{X}=$ $A^{f}(\theta p)^{-\varepsilon}$ und ermöglicht Exporterlöse von

$$
p k_{X}=p_{X} c_{X}=A^{f}(\theta p)^{1-\varepsilon} .
$$


Die Exporteure maximieren ihren Gewinn, indem sie wie in (2.10) einen Produzentenpreis $\alpha p=u$ als Aufschlag über die Kapitalnutzungskosten festsetzen. Der Exportgewinn beträgt $\pi_{X}=(1-t)(1-\alpha) p k_{X}$ bzw.

$$
\pi_{X}=(1-t) B^{f} /(\theta u)^{\varepsilon-1}, \quad B^{f}=\left(A^{f} / A\right) B .
$$

Anstatt den Auslandsmarkt mit Exporten zu beliefern, könnte dieselbe Unternehmung eine ausländische Produktionsstätte errichten. Die Produktion vor Ort spart Transportkosten, erfordert jedoch zusätzliche Fixkosten wegen der Duplikation der Produktion. Das Interesse richtet sich ausschliesslich auf die Auswirkungen der heimischen Gewinnsteuer auf den intensiven und extensiven Rand der Investitionen im Inland. Die Besteuerung im Ausland interessiert weiter nicht. Sie wird ganz vernachlässigt. Daher sind die Kapitalnutzungskosten im Ausland gleich dem Marktzins, $u^{f}=r$, der per Annahme dem heimischen Zins entspricht. Nachdem bei Direktinvestitionen und Produktion vor Ort keine Transportkosten anfallen, kann die Auslandstochter zu einem geringeren Preis $p_{I}$ an die ausländischen Nachfrager verkaufen. Der Wert der ausländischen Niederlassung für die heimische Muttergesellschaft beträgt

$$
\pi_{I}=\left(p_{I}-r\right) k_{I}
$$

Nachdem die Preiselastizität der Nachfrage im In- und Ausland identisch ist, wählt auch die ausländische Tochter einen Preisaufschlag wie in (2.10), $\alpha p_{I}=r$. Die Niederlassung erzielt einen Gewinn von $\pi_{I}=(1-\alpha) p_{I} k_{I}=B^{f} / r^{\varepsilon-1}$ mit $B^{f}$ wie in (2.15). Die Abwägung zwischen Exporten und Direktinvestitionen, wie sie in der Folge erläutert wird, ist nur dann wohl definiert, wenn $\pi_{I}>\pi_{X}$. Indem man die geschlossenen Lösungen vergleicht, erhält man die äquivalente Ungleichung $1 / r^{\varepsilon-1}>(1-t) /(\theta u)^{\varepsilon-1}$. Ohne Besteuerung mit $u=r$ wäre sie auf alle Fälle erfüllt. Mit positiven Handelskosten reduziert sich die Bedingung auf $1>1 / \theta^{\varepsilon-1}$ und ist damit notwendigerweise erfüllt, da $\varepsilon>1$ gilt. Wenn die Steuerunterschiede nicht allzugross werden, dann muss die Ungleichung auch für positive Steuersätze gelten. 


\subsection{Exporte Versus Direktinvestitionen}

Der Kern des Modells betrifft die Entscheidungen der heimischen Unternehmen, den Auslandsmarkt entweder mit Exporten oder durch Produktion vor Ort zu beliefern. ${ }^{4}$ Diese Entscheidung bestimmt den extensiven Rand der aggregierten Investitionen. Die Investitionen im Inland gehen zurück, wenn eine zunehmende Anzahl von Unternehmen die Produktion in das Ausland verlagert, weil Exporte relativ zu Direktinvestitionen an Attraktivität einbüssen. Der einfachste Ansatz ist, dass der Marktzutritt im Ausland mit Risiko behaftet ist und die Produkteinführung nur mit der Wahrscheinlichkeit $q$ gelingt. Alle Firmen mit einem neuen Produktdesign versuchen den Marktzutritt im Ausland, aber nur ein Teil ist tatsächlich erfolgreich, so dass es im Inland einen Teil von rein national operierenden Unternehmen gibt, die lediglich einen Gewinn von $\pi$ aus der Belieferung des Inlandsmarktes erwirtschaften. Dagegen beträgt der Gesamtgewinn von erfolgreichen Unternehmen aus ihrem globalen Absatz $\pi+\pi_{X}$ für die Exporteure und $\pi+\pi_{I}$ für die multinationalen Unternehmen mit ausländischen Niederlassungen. Ex ante, bevor der Erfolg des ausländischen Marktzutritts feststeht, wird mit einem erwarteten Gewinn aus dem weltweiten Absatz gerechnet,

$$
\bar{\pi}_{X}=\pi+q \pi_{X}, \quad \bar{\pi}_{I}=\pi+q \pi_{I} .
$$

Die Vorbereitung des Marktzutritts im Ausland verursacht Fixkosten wie z.B. für die Erfüllung von Regulierungen, den Aufbau eines Händlernetzes etc. Für manche der heimi-

\footnotetext{
${ }^{4}$ Wir endogenisieren diese Wahl mit einem sehr stark vereinfachten "Melitz-Modell" monopolistischer Konkurrenz (vgl. Melitz, 2003). Im Melitz-Modell unterscheiden sich die Firmen in ihrer Arbeitsproduktivität, so dass eine Verteilung von Einheitskosten, Preisen, Nachfrage und Firmengrössen resultiert. Dagegen schliesst der vorliegende Ansatz Produktivitätsunterschiede aus und erhält damit die Symmetrie in der Produktion und Nachfrage. Die einzige Form der Heterogenität liegt im Risiko des Marktzutritts im Ausland. Diese Annahmen reduzieren ganz erheblich die hohe Komplexität, die den analytischen Anwendungen des Melitz-Modells stark im Wege steht. Ein Nachteil des vorliegenden Ansatzes ist jedoch, dass jegliche Auswirkungen der Handels- und Finanzpolitik auf die aggregierte Produktivität, die aus einer Änderung in der Zusammensetzung des heterogenen Firmensektors folgen, ausgeschaltet werden. Diese Aspekte haben jedoch wenig mit dem Anliegen dieses Beitrags zu tun.
} 
schen Firmen mag sich daher der Export nicht mehr auszahlen, und schon gar nicht eine Direktinvestition. Die Fixkosten des Exports seien jedoch auf Null normalisiert, $f_{X}=0$, so dass alle Firmen zumindest den Export versuchen. Eine Entscheidung für Direktinvestitionen, um eine ausländische Produktionsgesellschaft zu errichten, ist jedoch teurer und ist daher nicht in allen Fällen profitabel. Die differentiellen Fixkosten der Direktinvestition seien $f_{I}$. Ex ante, bevor der Erfolg des Marktzutritts feststeht, ist der erwartete Firmenwert der Niederlassung, nach Abzug der Fixkosten, gleich $q \pi_{I} / R-f_{I}$. Aufgezinst auf die zweite Periode beträgt der Wert $q \pi_{I}-F$ mit $F \equiv R f_{I}$.

Die Unternehmen unterscheiden sich in der Wahrscheinlichkeit $q$, dass der Marktzutritt im Ausland erfolgreich ist. Mit der Wahrscheinlichkeit $1-q$ scheitert die Produkteinführung und es fallen überhaupt keine Erlöse an. Diese Wahrscheinlichkeit wird mit der Produktentwicklung aus einer Verteilung $G(q)$ gezogen. Je nach den Erfolgsaussichten $q$ entscheidet die Firma, ob sie den Auslandsmarkt mit Exporten oder mit Direktinvestitionen erschliessen will. Die zusätzlichen Fixkosten $F$ der Direktinvestition sind verloren, wenn die Produkteinführung misslingt. Daher rentieren sich Direktinvestitionen nur, wenn $\bar{\pi}_{I}-F>\bar{\pi}_{X}$. Diese Bedingung ist nur für jene Unternehmen erfüllt, die mit hoher Wahrscheinlichkeit erfolgreich sind und tatsächlich mit den geplanten Verkaufserlösen rechnen können. Unter allen Anbietern gibt es daher eine indifferente Firma mit einer kritischen Erfolgswahrscheinlichkeit $q$ :

$$
q\left(\pi_{I}-\pi_{X}\right)=F, \quad F \equiv f_{I} R
$$

Abbildung 2 illustriert die Abwägung zwischen Exporten und Direktinvestitionen. Da Exporte zusätzliche Transportkosten verursachen, sind die variablen Gewinne der Produktion vor Ort grösser, $\pi_{I}>\pi_{X}$, nachdem der Marktzutritt geschafft ist. Dagegen haben Direktinvestitionen höhere Fixkosten. Wenn eine Unternehmung nur mit einer geringen Erfolgswahrscheinlichkeit für den Marktzutritt rechnen kann, dann wird auch der differentielle Gewinn $\pi_{I}-\pi_{X}$ nur selten möglich, während die Fixkosten immer anfallen. ${ }^{5}$

\footnotetext{
${ }^{5}$ Solange $f_{I}>f_{X}$ erfüllt ist, gilt dasselbe Argument auch, wenn Exporte Fixkosten verursachen.
} 
Direktinvestitionen zahlen sich daher in diesem Fall nicht aus. Sie rentieren sich nur für Unternehmen mit hoher Erfolgswahrscheinlichkeit. Die Identität der kritischen Firma in der gesamten Verteilung teilt daher den Unternehmenssektor in Exporteure und multinationale Unternehmen mit ausländischen Niederlassungen auf.

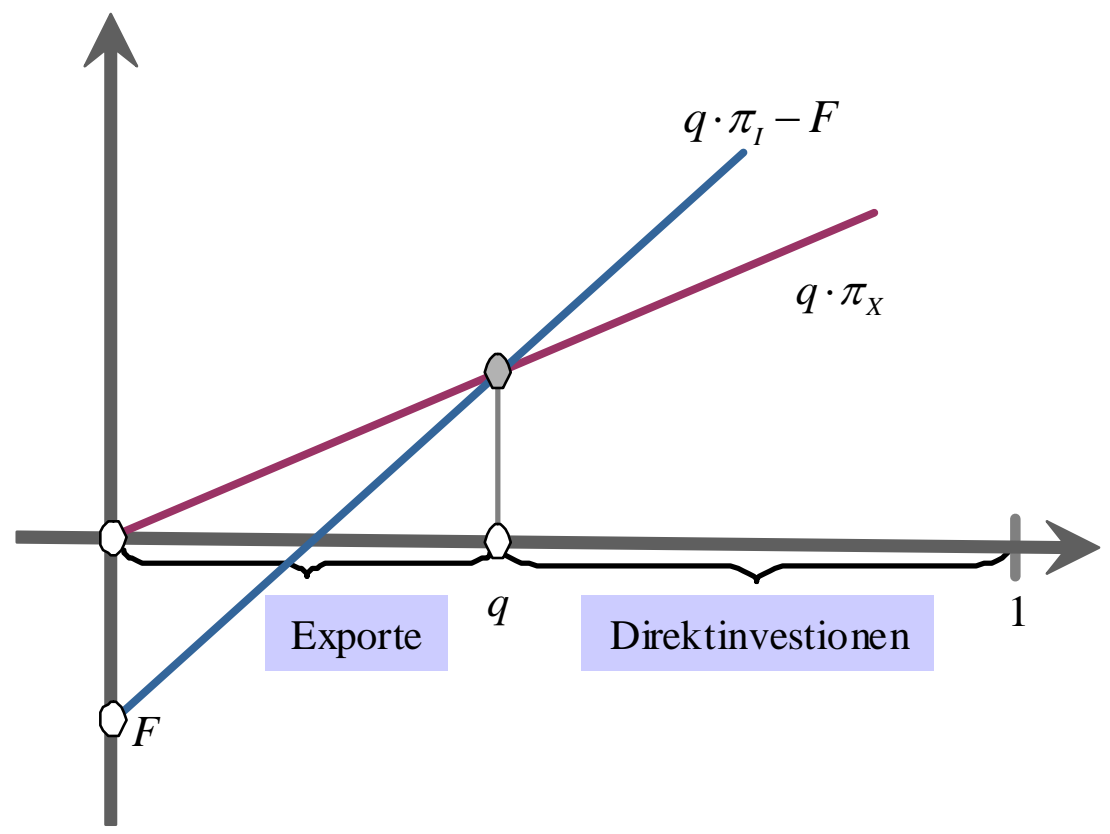

Abb. 2: Export versus Direktinvestition

Nach Abbildung 2 und Gleichung (2.18) wählen alle Anbieter mit eine Erfolgswahrscheinlichkeit $q^{\prime}<q$ Exporte, während der Rest den Weg der Direktinvestitionen einschlägt. Bei statistisch unabhängiger Verteilung beträgt im Aggregat der Anteil der Exporteure $s_{X}$ und jener der multinationalen Firmen $s_{I}$. Ein Anteil $1-s_{X}-s_{I}$ aller Unternehmen scheitert mit seiner Auslandsstrategie und beschränkt sich auf den inländischen Markt. Diese Unternehmen können daher das Wachstum aus der Erschliessung der Auslandsmärkte nicht realisieren und bleiben klein. Die Anteile sind

$$
s_{X}=\int_{0}^{q} q^{\prime} d G\left(q^{\prime}\right), \quad s_{I}=\int_{q}^{1} q^{\prime} d G\left(q^{\prime}\right), \quad s_{F}=\int_{q}^{1} d G\left(q^{\prime}\right) .
$$

Von allen $n$ Firmen müssen $n_{F}=s_{F} n$ die Fixkosten $F$ aufwenden, da sie den Marktzutritt über Direktinvestitionen versuchen. Der verbleibende Anteil $1-s_{F}$ wendet keine Fixkosten auf, da diese Firmen die Exportstrategie wählen. 
Ab nun sei die Gesamtanzahl der Firmen auf die Masse $n=1$ normalisiert. Daher bezeichnet $s_{X}$ sowohl die Zahl als auch den Anteil der Exporteure an allen Firmen. Dieselbe Interpretation gilt für $s_{I}$ und $s_{F}$. Im Aggregat beziehen daher die heimischen Haushalte monopolistische Gewinne im Gesamtwert von

$$
\pi^{e}=\pi+s_{X} \pi_{X}+\pi_{R}, \quad \pi_{R} \equiv \int_{q}^{1}\left(q \pi_{I}-F\right) d G\left(q^{\prime}\right)=s_{I} \pi_{I}-s_{F} F
$$

Dabei stammt der Betrag $\pi_{R}$ aus den repatriierten Gewinnen der ausländischen Niederlassungen nach Abzug der im Ausland aufgewendeten Fixkosten. Diese Gewinnüberweisungen sind Teil des Faktoreinkommens aus dem Ausland und erhöhen das heimische Bruttonationalprodukt im Vergleich zum Bruttoinlandsprodukt.

\subsection{Allgemeines Gleichgewicht}

Da wir lediglich die Mehrbelastung charakterisieren wollen, gehen wir davon aus, dass das gesamte Steueraufkommen in der zweiten Periode als Pauschaltransfer an die Haushalte zurückerstattet wird. International ist die Befreiungsmethode zur Beseitigung einer möglichen Doppelbesteuerung üblich, d.h. die Gewinne aus ausländischen Niederlassungen bleiben im Inland steuerfrei und sind nur mit ausländischer Gewinnsteuer belastet. Daher stammt des Gewinnsteueraufkommen nur aus den im Inland produzierenden Unternehmen. Die staatliche Budgetbeschränkung lautet

$$
z=t(p-e r)\left(k+s_{X} k_{X}\right)
$$

Die Ersparnisse des Haushaltssektors müssen alle im Inland getätigten Investitionen finanzieren, $S=K$. Diese Investitionen stellen Nachfrage nach dem Standardgut in der ersten Periode dar und reflektieren den Investitionsbedarf für den Inlandsabsatz und die im Inland produzierten Exporte,

$$
K \equiv k+s_{X} k_{X}
$$

Die Direktinvestitionen $s_{I} k_{I}$ der heimischen Konzerne im Ausland führen zu entsprechender Nachfrage nach dem Standardgut im Ausland. Die ausführlichere Version des Papiers 
in Keuschnigg (2006) zeigt Walras' Gesetz und führt die Markträumungsbedingungen und Handelsbilanzen der beiden Länder auf. Das hier präsentierte Modell ist ein einfaches allgemeines Gleichgewichtsmodell mit zwei Ländern und einem traditionellen und innovativen Sektor.

\section{Die Mehrbelastung der Gewinnsteuer}

\subsection{Effektive Steuersätze}

Zunächst ist zu klären, wie eine Änderung des tariflichen Gewinnsteuersatzes die effektiven Grenz- und Durchschnittssteuersätze (EMTR und EATR) verändert und wie diese Massgrössen die Investitionen am intensiven und extensiven Rand beeinflussen. Der Grenzsteuersatz misst die steuerliche Belastung eines marginalen Investitionsprojekts. Die Steuer treibt einen Keil zwischen die Ertragsrate $\alpha p=u$ vor Steuern und die Nettorendite $r$. Indem sie die notwendige Bruttorendite $u$ in die Höhe treibt, werden die marginalen Investitionen unrentabel. Die Steuer hemmt damit die intensive Kapitalbildung und das Unternehmenswachstum, wie Abbildung 1 veranschaulicht. Indem man den Steuerkeil als Anteil an der Vorsteuerrendite ausdrückt, erhält man den Grenzsteuersatz EMTR, der mit $t_{m}$ bezeichnet wird. Die Definition der Kapitalnutzungskosten in (2.9) ergibt

$$
t_{m} \equiv \frac{u-r}{u}=\frac{(1-e) t}{1-e t}, \quad 1-t_{m}=\frac{1-t}{1-e t} .
$$

Der Grenzsteuersatz verbindet die Brutto- und Nettorenditen mit $r=\left(1-t_{m}\right) u$ und fasst alle relevanten Informationen über die Ausgestaltung der Gewinnsteuer in einem einzigen Mass für die Verzerrung am intensiven Rand zusammen. Zum Beispiel würde eine Sofortabschreibung von Investitionen $(e=1)$ die Gewinnsteuer in eine Cash-Flow-Steuer mit einem Grenzsteuersatz von Null verwandeln. Die Steuer wäre neutral bezüglich der marginalen Investition, weil sie Kosten und Erträge der Investition zu gleichen Anteilen reduziert. Wenn keinerlei Investitionsausgaben abzugsfähig sind, $e=0$, dann fällt der Grenzsteuersatz mit dem tariflichen Satz zusammen, $t_{m}=t$. 
Der effektive Durchschnittssteuersatz (EATR) misst den Anteil der gesamten Steuerzahlung am Bruttoeinkommen. In einem intertemporalen Modell ist das relevante Konzept das Verhältnis des Barwerts der Steuern zum Barwert der Bruttogewinne. Nach (2.7) betragen die entsprechenden Werte in der zweiten Periode $\pi^{*} \equiv \pi+\pi^{T}=(p-r) k$ und $\pi^{T}=t(p-e r) k$. Die Definition des Durchschnittssatzes ist daher

$$
t_{a} \equiv \frac{\pi^{T}}{\pi^{*}}=\frac{p-e r}{p-r} t, \quad 1-t_{a}=\frac{\pi}{\pi^{*}}=(1-t) \frac{p-u}{p-r}
$$

Man beachte, dass wir von vollständiger Eigenkapitalfinanzierung ausgegangen sind und die Opportunitätskosten des Eigenkapitals bei der Gewinnsteuer nicht berücksichtigt sind. Die Kapitalkosten reduzieren aber den Bruttowert des Eigenkapitals $\pi^{*}$. Die Bemessungsgrundlage ist also breiter als der ökonomische Gewinn. Für den Fall $e<1$ ist daher der Durchschnittssatz grösser als der gesetzliche Satz, $t_{a}>t$. Bei vollständiger Abzugsfähigkeit der Investition fallen hingegen Durchschnitts- und tariflicher Satz zusammen, $t_{a}=t$, während der Grenzsteuersatz Null wird. Bruttogewinne, Nettogewinne und Steuerzahlungen hängen wie folgt zusammen: $\pi=\left(1-t_{a}\right) \pi^{*}$ und $\pi^{T}=t_{a} \pi^{*}$ mit $\pi^{*}=\pi+\pi^{T}$.

Um die komparativ statischen Effekte herauszuarbeiten, berechnen wir die relativen Änderungen im Vergleich zum Ausgangsgleichgewicht vor einer Steuerreform. Die Dachnotation macht relative Änderungen kenntlich, wie z.B. $\hat{u} \equiv d u / u$. Die Ausnahmen sind Änderungen in den Steuersätzen, die relativ zu den ursprünglichen Nettosteuerpreisen ausgedrückt werden, z.B. $\hat{t}_{m} \equiv d t_{m} /\left(1-t_{m}\right)$. Da der Preisaufschlag in (2.10) konstant bleibt, ändern sich Produzentenpreise und Kapitalnutzungskosten $u=r /\left(1-t_{m}\right)$ im selben Verhältnis wie der Grenzsteuersatz,

$$
\hat{p}=\hat{u}=\hat{t}_{m}
$$

Wie ändern sich die effektiven Steuersätze mit einer Anpassung des tariflichen Satzes? Man beachte, dass die Durchschnittsbelastung EATR ein endogenes Mass ist, welches nur gemeinsam mit der Auswirkung der Steuer auf das Gleichgewicht bestimmt werden kann. Die relative Änderung folgt aus der Log-Linearisierung der Gleichung für $1-t_{a}$ in 
(3.2) mit dem Ergebnis $-\hat{t}_{a}=-\hat{t}+\frac{d p-d u}{p-u}-\frac{d p}{p-r}$. Indem man geeignet erweitert und (3.3) berücksichtigt, folgt

$$
\hat{t}_{a}=\hat{t}+\frac{r}{p-r} \hat{t}_{m}, \quad \hat{t}_{m}=\frac{1-e}{1-e t} \hat{t} .
$$

Eine erste Einsicht ist, dass der gesetzliche Steuersatz die Durchschnittsbelastung einerseits direkt und andererseits indirekt über den Einfluss auf den Grenzsteuersatz bestimmt. Eine Cash-Flow-Steuer mit Sofortabschreibung reduziert den Grenzsteuersatz auf Null. Die Durchschnittsbelastung der Cash-Flow-Steuer ist identisch mit dem tariflichen Satz, $\hat{t}_{m}=0$ und $\hat{t}_{a}=\hat{t}$. Obwohl sie neutral bezüglich der intensiven Investition ist, verzerrt sie weiterhin am extensiven Rand der Investitionstätigkeit.

\subsection{Investition und Gewinne}

Der Grenzsteuersatz EMTR drückt die Kapitalnutzungskosten hoch, so dass die monopolistischen Anbieter nach der gewinnmaximierenden Aufschlagsregel einen höheren Preis setzen und eine geringere Absatzmenge wählen. Dementsprechend investieren sie weniger. Aus der Nachfragekurve in (2.4) folgt

$$
\hat{k}=-\varepsilon \hat{u}=-\varepsilon \hat{t}_{m}
$$

Der Gewinn nach Steuern hängt von den Durchschnitts- und Grenzsteuersätzen ab. Bei einem Bruttogewinn von $\pi^{*}=(p-r) k$ bleibt ein Gewinn nach Steuer von $\pi=$ $\left(1-t_{a}\right) \pi^{*}$. Die Anpassung des Bruttogewinns spiegelt die Preiserhöhung und die geringere Produktion wider. In log-linearisierter Form ergibt sich $\hat{\pi}^{*}=\frac{p}{p-r} \hat{p}+\hat{k}$ bzw.

$$
\hat{\pi}=\hat{\pi}^{*}-\hat{t}_{a}=-\left(\varepsilon-\frac{p}{p-r}\right) \hat{t}_{m}-\hat{t}_{a}=-\frac{p-e r}{p-u} \hat{t}, \quad \hat{\pi}_{X}=\hat{\pi} .
$$

Die dritte Gleichung zeigt den Nettoeffekt aus der Anpassung des tariflichen Satzes auf. Alternativ erhält man das Ergebnis direkt aus der Anwendung des Envelopentheorems auf (2.7), $d \pi / d t=-(p-e r) k$ und der anschliessenden Division mit $\pi=(1-t)(p-u) k .^{6}$

\footnotetext{
${ }^{6}$ Die beiden Ausdrücke sind äquivalent. Verwende $\alpha=(\varepsilon-1) / \varepsilon$ in der Bedingung erster Ordnung
} 
Eine Cash-Flow-Steuer mit $e=1$ vermeidet eine Verzerrung des intensiven Investitionskalküls und führt zu $t_{m}=0$ und $t_{a}=t$. Eine Zunahme des tariflichen Satzes hätte dann keinen Einfluss auf den Bruttogewinn, $\hat{\pi}^{*}=0$, würde aber den Nettogewinn um $\hat{\pi}=-\hat{t}_{a}=-\hat{t}$ mindern.

Unter sonst gleichen Umständen reduziert ein Anstieg des tariflichen Steuersatzes die Gewinne aus dem Export in genau derselben Weise. Auch wenn das Nachfrage- und Produktionsvolumen sich unterscheiden, so bleibt dennoch die relative Änderung der Nettogewinne gleich, da die Nachfrageelastizitäten auf den heimischen und ausländischen Märkten per Annahme identisch sind. Unter der Annahme, dass das Inland zur Vermeidung einer Doppelbesteuerung von Auslandsgewinnen die Befreiungsmethode anwendet, werden die Auslandsgewinne $\pi_{I}$ aus Direktinvestitionen nicht mehr weiter im Inland besteuert, wie auch aus (2.16) hervorgeht. Die Investitionen der Niederlassungen im Ausland hängen nur von den ausländischen Kapitalnutzungskosten ab, die möglicherweise mit ausländischen Steuern an der Quelle belastet sind.

Abbildung 2 illustriert die Abwägung zwichen Export und Direktinvestition, die mit dem kritischen Wert $q$ in (2.18) formal charakterisiert ist. Die Log-Linearisierung ergibt $\hat{q}=\hat{\pi}_{X} \pi_{X} /\left(\pi_{I}-\pi_{X}\right)$, da die Höhe der Auslandsgewinne $\pi_{I}$ aus der Perspektive des Inlands exogen ist. Nach Einsetzen der Veränderung der Exportgewinne erhält man

$$
\hat{q}=\frac{\pi_{X}}{\pi_{I}-\pi_{X}} \hat{\pi}, \quad \hat{\pi}=-\frac{p-e r}{p-u} \hat{t} .
$$

Die heimische Gewinnsteuer führt aus zwei Gründen zu einem verstärkten Abfluss von Direktinvestitionen. Erstens hebt sie die Durchschnittsbelastung an und reduziert somit die Nettogewinne aus dem Exportgeschäft, so dass der ausländische Marktzutritt mittels Direktinvestition attraktiver wird. Zweitens führt sie im Normalfall auch zu einer höheren Grenzsteuerbelastung. Damit beeinträchtigt die Steuer Investitionen und Unternehmenswachstum und reduziert damit ebenfalls die Exportgewinne relativ zu den Auslandsge(2.10) und erhalte $\varepsilon=\frac{p}{p-u}$. Nach Einsetzen in die runde Klammer in (3.6) folgt zusammen mit $\hat{t}_{a}$ aus (3.4) $\hat{\pi}=-\frac{u}{p-u} \hat{t}_{m}-\hat{t}$. Im letzten Schritt ersetzt man $\hat{t}_{m}=\frac{1-e}{1-e t} \hat{t}$, verwendet $u=\frac{1-e t}{1-t} r$ aus (2.9) und erhält nach einigen Umformungen $\hat{\pi}=\frac{p-e r}{p-u} \hat{t}$. 
winnen aus Direktinvestitionen. Der Nettoeffekt ist in (3.6) aufgeführt und benachteiligt damit die Investitionen in die heimische Exportproduktion relativ zu den Direktinvestitionen im Ausland. Indem die Steuer den kritischen Wert für die Erfolgswahrscheinlichkeit des Marktzutritts mindert, steigert sie die Attraktivität von Direktinvestitionen und mindert die Zahl der im Inland produzierenden Exportunternehmen. Je mehr Firmen sich dazu entscheiden, die Produktion ins Ausland zu verlagern und den Markt aus lokaler Produktion zu versorgen, desto mehr verschiebt sich die Zusammensetzung des Firmensektors von den Exporteuren zu den multinationalen Unternehmen. Die Log-Linearisierung von (2.19) zeigt formal die Veränderung der Unternehmensanteile,

$$
\hat{s}_{X}=\mu_{X} \hat{q}, \quad \hat{s}_{I}=-\mu_{I} \hat{q}, \quad \hat{s}_{F}=-\mu_{F} \hat{q}
$$

wobei die Koeffizienten $\mu_{X} \equiv q^{2} g(q) / s_{X}, \mu_{I} \equiv q^{2} g(q) / s_{I}$ und $\mu_{F} \equiv q g(q) / s_{F}$ positiv definiert sind.

Die Gewinne der Exporteure und multinationalen Unternehmen unterscheiden sich, da nur die Exporteure Handelskosten aufwenden und damit höhere Preise verlangen müssen. Dies schmälert Absatz und Gewinn. Die Gewinnsteuer wird daher das aggregierte Gewinneinkommen $\pi^{e}$ nach Steuern nicht nur wegen der Minderung der Exportgewinne, sondern auch wegen der Auswirkung auf die Zusammensetzung des Unternehmenssektors beeinflussen. Nach (3.8) wirkt sich eine Änderung der kritischen Erfolgswahrscheinlichkeit mit $d s_{X}=q g(q) d q=-d s_{I}$ auf die Firmenstruktur aus. Daher passt sich der erwartete Gewinn in (2.20) mit $\pi^{e} \hat{\pi}^{e}=\pi \hat{\pi}+s_{X} \pi_{X} \hat{\pi}_{X}-\left[q\left(\pi_{I}-\pi_{X}\right)-F\right] g(q) d q$ an. Der eckige Klammerausdruck verschwindet als Folge der endogenen Exportentscheidung. Indem man für die Änderung der Gewinne (3.6) einsetzt, erhält man

$$
\pi^{e} \hat{\pi}^{e}=-\left(\pi+s_{X} \pi_{X}\right) \frac{p-e r}{p-u} \hat{t} .
$$

\subsection{Mehrbelastung}

Die Mehrbelastung der Gewinnsteuer ist ein Ausdruck dessen, dass die Belastung des privaten Sektors gemessen am verursachten Wohlfahrtsverlust das vom Staat erzielte Steuer- 
aufkommen übersteigt. Um den Nettoverlust zu ermitteln, definiere man die Steuerbasis bzw. Bemessungsgrundlage $B$ und schreibe die Steuereinnahmen als

$$
z=t B, \quad B \equiv(p-e r) K, \quad K \equiv k+s_{X} k_{X}
$$

Die Ergiebigkeit der Steuer wird dadurch gemindert, dass sie die Investitionen reduziert. Die Elastizitäten $\sigma$ and $\eta$ bestimmen, wie stark tatsächlich die Steuerbasis am intensiven und extensiven Rand schrumpft. Die intensive Elastizität $\sigma$ misst, wie die Beeinträchtigung der intensiven Investition aufgrund einer höheren Grenzsteuerbelastung EMTR die Steuerbasis aushöhlt, wenn der extensive Rand festgehalten wird, $\hat{s}_{X}=0$. Die Anpassung der Steuerbasis in (3.10) beträgt $\hat{B}=\frac{p}{p-e r} \hat{p}+\hat{K}$. Indem man $\hat{p}=\hat{t}_{m}, \hat{K}=-\varepsilon \hat{t}_{m}$ und $\hat{t}_{m}=\frac{1-e}{1-e t} \hat{t}$ einsetzt, folgt

$$
\hat{B}=-\sigma \hat{t}, \quad \sigma \equiv-\left(\varepsilon-\frac{p}{p-e r}\right) \frac{1-e}{1-e t} .
$$

Die extensive Elastizität $\eta$ des aggregierten Kapitalstocks bezüglich des Gewinndifferentials $\pi_{I}-\pi_{X}$ spiegelt den Einfluss auf den Anteil $s_{X}$ der Exporteure wider. Indem man (3.10) log-linearisiert und alle Ausdrücke sammelt, die sich auf die Änderung von $s_{X}$ beziehen, erhält man

$$
\hat{K}=\eta \hat{\pi}_{X}, \quad \eta \equiv \frac{s_{X} k_{X}}{K} \frac{\mu_{X} \pi_{X}}{\pi_{I}-\pi_{X}} .
$$

Die Steuerbasis reagiert am extensiven Rand mit $\hat{B}=\hat{K}=-\eta \frac{p-e r}{p-u} \hat{t}$. Man benutze $(p-e r) k_{X}=B_{X}, t B_{X}=t_{a} \pi_{X}^{*}$ als auch $(1-t)(p-u) k_{X}=\pi_{X}=\left(1-t_{a}\right) \pi_{X}^{*}, \mathrm{um}$ $\frac{(p-e r) k_{X}}{(p-u) k_{X}}=\frac{t_{a}}{1-t_{a}} \frac{1-t}{t}$ zu schreiben. Man erhält $\hat{B}=-\eta \frac{t_{a}}{1-t_{a}} \frac{1-t}{t} \hat{t}$. Nach diesen Zwischenschritten folgt als Anpassung der Steuerbasis $d z=(1-t) B\left[\hat{t}+\frac{t}{1-t} \hat{B}\right]$ bzw.

$$
d z=(1-t) B\left[1-\frac{t}{1-t} \sigma-\frac{t_{a}}{1-t_{a}} \eta\right] \hat{t}
$$

Der erste Term in der eckigen Klammer steht für den direkten Aufkommenseffekt aus der Anhebung des tariflichen Steuersatzes. Der zweite Ausdruck mit der Elastizität $\sigma$ misst den verzerrenden Effekt der Steuer auf die intensive Investitionsneigung. Dies hat zwei Konsequenzen für die Steuerbasis. Einerseits schrumpft die Steuerbasis, weil das gebremste Unternehmenswachstum die Gewinne mindert. Andererseits nimmt die Steuerbasis zu, 
weil bei geringerem Output die Preise und damit die Gewinne steigen. Der dritte Term mit der Elastizität $\eta$ resultiert aus dem wertmindernden Effekt eines höheren Steuersatzes, der eine extensive Anpassung der Investition und damit eine Produktionsverlagerung ins Ausland auslöst.

Dem Anstieg des Steueraufkommens in (3.13) ist die Wohlfahrtseinbusse des privaten Sektors gegenüberzustellen. Diese ergibt sich aus dem Differential von $(2.3), d V=$ $\pi^{e} \hat{\pi}^{e}+d z-c d p$. Der letzte Teil steht für den Verlust an Konsumentenrente, wenn der Preis angehoben wird, wie man sich leicht anhand von Abbildung 1 klar macht. Man beachte zunächst, dass Nettogewinne und Steuerbasis $B=(p-e r) K$ wie folgt miteinander zusammenhängen,

$$
\pi+s_{X} \pi_{X}=(1-t)(p-u) K=(1-t) B \frac{p-u}{p-e r} .
$$

Daher ermittelt man für die erwarteten privaten Gewinne in $(3.9) \pi^{e} \hat{\pi}^{e}=-(1-t) B \hat{t}$. Eingesetzt in die vorhin abgeleitete Wohlfahrtsformel folgt zusammen mit (3.13)

$$
d V=-(1-t) B\left[\frac{t}{1-t} \sigma+\frac{t_{a}}{1-t_{a}} \eta+\Omega\right] \hat{t}, \quad \Omega \equiv \frac{(p-u) \varepsilon k}{(1-t) B} \frac{1-e}{1-e t} .
$$

Der Term $\Omega$ drückt den Verlust der Konsumentenrente $c d p$ als Folge der Preiserhöhung aus. Der Preisanstieg ist eine Folge der steuerlich induzierten Einschränkung der Outputmenge $c=k$ und beträgt $\hat{p}=-\frac{1}{\varepsilon} \hat{k}$ bzw. $c d p=-\frac{1}{\varepsilon} p d k$. Einsetzen von $\frac{1}{\varepsilon}=1-\alpha=\frac{p-u}{p}$ aus der Preisaufschlagsregel ergibt $c d p=-(p-u) d k$. Der Verlust an Konsumentenrente entspricht daher dem Keil $p-u$ zwischen Preis und Grenzkosten multipliziert mit der Mengenreduktion $d c=d k$ im Konsum. Im Gleichgewicht resultiert aus (3.3-3.5) ein Outputund Konsumverlust von $d k=-k \varepsilon \frac{1-e}{1-e t} \hat{t}$. Die Kombination dieser Schritte ergibt die Definition von $\Omega$. Dieser Ausdruck steht für die Mehrbelastung, die aus der zusätzlichen Verzerrung des Preisaufschlags als Folge der geringeren intensiven Investition entsteht.

Wir können nun die marginale Mehrbelastung der Gewinnsteuer bzw. Körperschaftssteuer ermitteln, indem wir den zusätzlichen Wohlfahrtsverlust in (3.15) durch das zusätzliche Steueraufkommen in (3.13) dividieren,

$$
M D W L=-\frac{d V}{d z}=\frac{\frac{t}{1-t} \sigma+\frac{t_{a}}{1-t_{a}} \eta+\Omega}{1-\frac{t}{1-t} \sigma-\frac{t_{a}}{1-t_{a}} \eta}
$$


Die volkswirtschaftlichen Grenzkosten der Besteuerung (marginal costs of public funds) sind als eins plus die marginale Mehrbelastung definiert und betragen

$$
M C P F=\frac{1+\Omega}{1-\frac{t}{1-t} \sigma-\frac{t_{a}}{1-t_{a}} \eta} .
$$

Abgesehen vom zusätzlichen Term $\Omega$, der sich auf die Verzerrung des Preisaufschlags bezieht, ist dieses Ergebnis fast vollständig identisch mit der Formel, die sich aus der Analyse der intensiven und extensiven Arbeitsangebotsverzerrung ergibt. Man vergleiche dazu bespielsweise Keuschnigg (2005), der in Gleichung (III.34) die Ergebnisse von Immervoll, Kleven, Kreiner und Saez (2007) und Kleven und Kreiner (2006) aufbauend auf eine frühere Arbeit von Saez (2002) herleitet.

Die Betrachtung von zwei Spezialfällen hilft, die Implikationen dieses Ergebnisses vollständig zu erschliessen. Zuerst sei angenommen, dass die zusätzlichen Fixkosten von Direktinvestitionen so prohibitiv hoch sind, dass Investitionen im Ausland vollständig unterbunden sind. Daher ist der Anteil $s_{X}$ der erfolgreichen Exporteure fix (und $s_{I}=s_{F}=0$ in 2.19), was die Investitionsreaktion am extensiven Rand unterdrückt, $\eta=0$. Es bleibt ausschliesslich die übliche Verzerrung der marginalen Investitionen im Inland übrig,

$$
M C P F=\frac{1+\Omega}{1-\frac{t}{1-t} \sigma} .
$$

In diesem Fall wäre die Cash-Flow-Steuer mit Sofortabschreibung von Investitionen $(e=$ 1) vollständig neutral. Sowohl $\sigma$ als auch $\Omega$ wären Null. Die Steuer hätte weder einen Einfluss auf das Unternehmenswachstum noch würde sie die Konsumentenrente wegen eines noch höheren Preisaufschlags beeinträchtigen. Die Grenzkosten der Besteuerung wären wie bei einer Pauschalsteuer gleich eins.

Ein zweites interessantes Szenario ist die Erhöhung einer Cash-Flow-Steuer $(e=1)$, ohne die extensive Investitionsreaktion zu unterdrücken. Die Grenzsteuerbelastung ist dann vor und nach der Intervention gleich Null. Die Steuer vermeidet vollständig die Verzerrung der intensiven Investition. Die Grenzkosten der Besteuerung spiegeln nur mehr die extensive Investitionsreaktion wider,

$$
M C P F=\frac{1}{1-\frac{t_{a}}{1-t_{a}} \eta} .
$$


Die Cash-Flow-Steuer ist in einer Wirtschaft mit multinationalen Direktinvestitionen nicht neutral, weil sie nach wie vor inframarginale Renten besteuert und damit weiterhin die Standortentscheidungen verzerrt. Das Ausmass der Verzerrung und damit die Höhe der Grenzkosten der Besteuerung hängen von der Durchschnittsbelastung $t_{a}$ und der extensiven Elastizität $\eta \mathrm{ab}$. Die Elastizität ist in (3.12) definiert und misst, wie stark die aggregierte Investition $K$ zurückgeht, wenn mehr Firmen die Produktion ins Ausland verlagern und dort investieren.

\section{Schlussfolgerungen}

In der finanzwissenschaftlichen Literatur fehlt bisher eine Charakterisierung der Mehrbelastung der Gewinnsteuer in einem konsistenten Rahmen, der die gleichzeitige Auswirkung der Steuer auf die intensive und extensive Investitionsneigung berücksichtigen würde. Die Steuer beeinträchtigt eben nicht nur das Unternehmenswachstum, sondern auch die Standortentscheidungen. Diese Lücke ist umso gravierender, als die neuere steuerpolitische Diskussion nicht nur der Grenzsteuerbelastung, sondern vor allem der effektiven Durchschnittsbelastung der Unternehmen im internationalen Vergleich eine grosse Bedeutung für gesamtwirtschaftliche Investitionen und Wachstum beimisst (vgl. z.B. Sachverständigenrat et al., 2006, oder Europäische Kommission, 2001). Das Gutachten des Sachverständigenrates weist die Auswirkungen seines Reformvorschlags auf die effektive Grenzsteuerbelastung gar nicht mehr separat aus, sondern listet lediglich die Veränderung der Kapitalnutzungskosten auf. Dagegen betont es sehr stark die Verringerung der Durchschnittsbelastung in Deutschland relativ zu wichtigen Vergleichsländern, um die Vorteile des Reformvorschlags für die Standortqualität Deutschlands bezüglich internationaler Direktinvestitionen hervorzuheben.

Die traditionelle Sicht ist geprägt von der Auswirkung der Gewinnsteuer auf marginale Investitionsprojekte und den damit einhergehenden Wachstumseinbussen. Die traditionelle Einschätzung der volkswirtschaftlichen Grenzkosten der Gewinnsteuer muss vermutlich 
ganz erheblich nach oben korrigiert werden, da die Steuer die aggregierte Investition auf zweifache Weise sowohl am intensiven als auch am extensiven Rand beeinträchtigt. Erstens investieren alle im Inland etablierten Unternehmen weniger. Zweitens wird ein Teil der Unternehmen die Produktion für ausländische Märkte nicht mehr im Inland ansiedeln, sondern in das Ausland verlegen. Die Mehrbelastung der Gewinnsteuer hängt dann zu einem guten Teil von der effektiven Durchschnittsbelastung und der Höhe der extensiven Investitionselastizität ab. Diese Elastizität bestimmt, wie viele der Produktionsstätten in das Ausland verlagert werden, wenn die Steuer den Unterschied des möglichen Nettogewinns weiter vergrössert und so die steuerliche Standortqualität beeinträchtigt.

\section{Literatur}

[1] Bond, Stephen R. (2000), Levelling Up or Levelling Down? Some Reflections on the ACE and CBIT Proposals, and the Future of the Corporate Tax Base, in: Sijbren Cnossen (Hrsg.), Taxing Capital Income in the European Union, Oxford: Oxford University Press, 161-179.

[2] Davies, Ronald B. (2004), Tax Treaties and Foreign Direct Investment: Potential versus Performance, International Tax and Public Finance 11, 775-802.

[3] Devereux, Michael P. und Rachel Griffith (1998), Taxes and the Location of Production: Evidence from a Panel of US Multinational Firms, Journal of Public Economics $68,335-367$.

[4] Devereux, Michael P. und Rachel Griffith (2003), Evaluating Tax Policy for Location Decisions, International Tax and Public Finance 10, 107-126.

[5] Devereux, Michael P., Rachel Griffith und Alexander Klemm (2002), Corporate Income Tax Reforms and International Tax Competition, Economic Policy 17, 451-495.

[6] Devereux, Michael P. und Glenn R. Hubbard (2003), Taxing Multinationals, International Tax and Public Finance 10, 469-487.

[7] Europäische Kommission (2001), Company Taxation in the Internal Market, Commission Staff Working Paper, COM(2001)582 Final.

[8] Fuest, Clemens (2005), Economic Integration and Tax Policy With Endogenous Foreign Firm Ownership, Journal of Public Economics 89, 1823-1840. 
[9] Gordon, Roger H. und James R. Hines Jr. (2002), International Taxation, in: Alan J. Auerbach und Martin Feldstein (Hrsg.), Handbook of Public Economics, Vol. 4, Amsterdam: Elsevier, 1935-1995.

[10] Gresik, Thomas A. (2001), The Taxing Task of Taxing Transnationals, Journal of Economic Literature 39, 800-838.

[11] Grossman, Gene und Elhanan Helpman (2005), Outsourcing in a Global Economy, Review of Economic Studies 72, 135-159.

[12] Haufler, Andreas und Guttorm Schjelderup (2000), Corporate Tax Systems and Cross Country Profit Shifting, Oxford Economic Papers 52, 306-325.

[13] Helpman, Elhanan (2006), Trade, FDI, and the Organization of Firms, Journal of Economic Literature 44, 589-630

[14] Helpman, Elhanan, Marc J. Melitz und Stephen R. Yeaple (2004), Export versus FDI with Heterogeneous Firms, American Economic Review 99, 300-316.

[15] Immervoll, Herwig, Henrik Kleven, Claus Thustrup Kreiner und Emmanuel Saez (2007), Welfare Reform in European Countries: A Microsimulation Analysis, Economic Journal 117, 1-44.

[16] Janeba, Eckhard (1997), International Tax Competition, Tübingen: Mohr Siebeck.

[17] Keuschnigg, Christian (2004), Eine Einkommensteuerreform für mehr Wachstum in der Schweiz, Zürich: Avenir Suisse.

[18] Keuschnigg, Christian (2005), Öffentliche Finanzen: Einnahmenpolitik, Tübingen: Mohr Siebeck.

[19] Keuschnigg, Christian (2006), Corporate Taxation, Exports and Foreign Direct Investment, Universität St. Gallen, www.iff.unisg.ch, Seite Forschung/DP Finanzwissenschaft, und CEPR DP 5769.

[20] Keuschnigg, Christian und Martin D. Dietz (2007), A Growth Oriented Dual Income Tax, International Tax and Public Finance 14, 191-221.

[21] Kleven, Henrik und Claus Thustrup Kreiner (2006), The Marginal Cost of Public Funds: Hours of Work Versus Labor Force Participation, Journal of Public Economics 90, 1955-1973.

[22] Melitz, Marc J. (2003), The Impact of Trade on Intra-Industry Reallocations and Aggregate Industry Productivity, Econometrica 71, 1695-1725.

[23] President's Advisory Panel on Federal Tax Reform (2006), Simple, Fair, and ProGrowth: Proposals to Fix America's Tax System, Washington, D.C. 
[24] Sachverständigenrat, Max-Planck-Institut für Geistiges Eigentum, Wettbewerbs- und Steuerrecht, und Zentrum für Europäische Wirtschaftsforschung (2006), Reform der Einkommens- und Unternehmensbesteuerung durch die Duale Einkommensteuer, Wiesbaden: Statistisches Bundesamt.

[25] Saez, Emmanuel (2002), Optimal Income Transfer Programs: Intensive Versus Extensive Labor Supply Responses, Quarterly Journal of Economics 117, 1039-1073.

[26] Sørensen, Peter B., Hrsg., (2004), Measuring the Tax Burden on Capital and Labor, CESifo Seminar Series, Cambridge: MIT Press.

[27] Weichenrieder, Alfons J. (1995), Besteuerung und Direktinvestition, Tübingen: Mohr Siebeck.

[28] Wissenschaftlicher Beirat beim Bundesministerium der Finanzen (2004), Flat Tax oder Duale Einkommensteuer? Zwei Entwürfe zur Reform der deutschen Einkommensbesteuerung, BMF-Schriftenreihe 78, Berlin. 